\title{
Brain Metastasis with High Protein Content- An Extremely Rare Imaging Feature
}

\author{
G Lakshmi Prasad ${ }^{1 *}$, Rajesh Nair $^{1 *}$ and Geetha Vasudevan ${ }^{2}$
}

${ }^{1}$ Department of Neurosurgery, Kasturba Medical College, Manipal University, Manipal, Karnataka, India ${ }^{2}$ Department of Pathology, Kasturba Medical College, Manipal University, Manipal, Karnataka, India

\begin{abstract}
Metastasis forms the most common brain malignancy. Solitary or multiple ring enhancing intra-axial lesions bordered on the gray-white matter junction with disproportionate edema is the usual pattern on imaging. No reports of high protein content parenchymal metastatic lesion can be found in literature till now. Authors here describe a case of 42 year old female presenting with features of intracranial hypertension and imaging suggestive of a high protein containing lesion. Gross total resection was performed and histopathological features were suggestive of metastatic papillary adenocarcinoma.
\end{abstract}

Keywords: T1 hyperintense lesions; Metastatic papillary adenocarcinoma; High protein content

\section{Introduction}

Metastasis represents the most common brain malignancy. Solitary or multiple ring enhancing intra-axial lesions bordered on the graywhite matter junction with disproportionate edema is the usual pattern on imaging. No reports of high protein content parenchymal metastatic lesion can be found in literature till now. Authors here describe a case of 42 year old female presenting with features of intracranial hypertension and imaging suggestive of a high protein containing lesion. Gross total resection was performed and histopathological features were suggestive of metastatic papillary adenocarcinoma.

\section{Case Description}

A 42-year old woman without past medical history, presented to our clinic with a three-month history of holocranial headache and one week history of blurring of vision. Neurological examination revealed homonymous hemianopia with papilledema. A thyroid nodule was noted on general examination. Breast examination was normal. Biochemical examination showed evidence of mild hypothyroidism while tumour markers (CA 19.9, CA 125 and CEA) were within normal limits. Fine needle aspiration cytology (FNAC) from the thyroid nodule, preoperatively, featured a diagnosis of follicular neoplasm. CT brain showed features of a homogeneously hyperdense intra-axial lesion in the right occipital lobe with peri lesional edema and mass effect (Figure 1a). On MRI, it appeared to be consisting of 2 components- peripheral component of the lesion being hyper intense on T1, T2 weighted and FLAIR sequences and inner component being isointense on all these sequences (Figure $1 \mathrm{~b}(\mathrm{a}-\mathrm{d})$ ). The inner component showed restriction on diffusion weighted images and also mild enhancement on contrast images (Figure $1 \mathrm{~b}$ (e and f). On MR spectroscopy (MRS), there was low intratumoral Cho/Cr ratio with perilesional low NAA/Cho ratio (Figure 2). There was no fluid level noted. Based on these imaging patterns, radiologically, features were suggestive of a high protein-containing lesion. A differential diagnosis of metastasis or an ependymoma was thought of, based on the location. A thorough clinical and radiological work-up (Tumor markers, Chest X-ray, Peripheral smear, Ultrasound abdomen and PET Scan) for the primary lesion was negative. Intraoperatively, the tumor was heterogeneous, was reaching up the surface and possessing solid and cystic components. The cyst contained dark, thick, viscid fluid, possessing a "machinery oil" like consistency. The solid component was moderately vascular, soft to firm in consistency and "easily suckable" [1]. Gross total resection was performed.
Histopathology revealed nuclear atypia within malignant columnar cells arranged over a papillary fibrovascular core, features suggestive of metastatic papillary adenocarcinoma (Figure 3). Cytological examination of the proteinacious cyst content was also positive for malignancy. She was further referred for whole brain radiotherapy.

\section{Discussion}

Metastases represents the most common brain neoplasm worldwide [2-4]. They are usually multiple and well-demarcated with disproportionate surrounding edema. Cerebral hemispheres are the most common location followed by cerebellum. [2,5]. This is dictated by the size of the region and the proportion of blood flow $[2,6]$. They are usually located at the gray-white matter junction in water-shed areas $[2,4,5]$. Lung, breast, melanoma and renal carcinoma are the most common primary sites of brain metastases. [2,4]. The advent of modern imaging techniques such as MRI has tremendously improved the diagnosis of intracranial lesions in recent years. The appearance of these lesions on various MR sequences holds the key to such diagnoses [7]. On imaging, metastatic lesions usually appear hypointense on T1 and hyperintense on $\mathrm{T} 2$ weighted images (WI) with varying patterns of enhancement, ranging from homogeneous to a ring pattern [8]. The main differential diagnosis include, abscess, high grade gliomas such as glioblastoma multiforme (GBM), CNS lymphomas, demyelinating and inflammatory lesions [2,8]. Additional sequences such as MR spectroscopy (MRS), MR perfusion (MRP) and diffusion images also assist in closing onto the diagnosis of such lesions [8,9]. Although, tumoral MRS and MRP findings are often equivocal for mets and GBM, evaluation of the peritumoral T2 hyperintense edema by MRS, MRP, DWI provide the best means of differentiating between primary high grade tumours and solitary metastasis [8].

*Corresponding author: Lakshmi Prasad G, Rajesh Nair, Department of Neurosurgery, Kasturba Medical College, Manipal University, Manipal, Karnataka, India, Tel: 0091-9611519911; E-mail: rajeshnair39@yahoo.com

Received January 07, 2015; Accepted January 28, 2015; Published January 30 2015

Citation: Prasad GL, Nair R, Vasudevan G (2015) Brain Metastasis with High Protein Content- An Extremely Rare Imaging Feature. J Neurol Disord 3: 208. doi:10.4172/2329-6895.1000208

Copyright: ( 2015 Prasad GL, et al. This is an open-access article distributed under the terms of the Creative Commons Attribution License, which permits unrestricted use, distribution, and reproduction in any medium, provided the original author and source are credited. 
Citation: Prasad GL, Nair R, Vasudevan G (2015) Brain Metastasis with High Protein Content- An Extremely Rare Imaging Feature. J Neurol Disord 3: 208. doi:10.4172/2329-6895.1000208

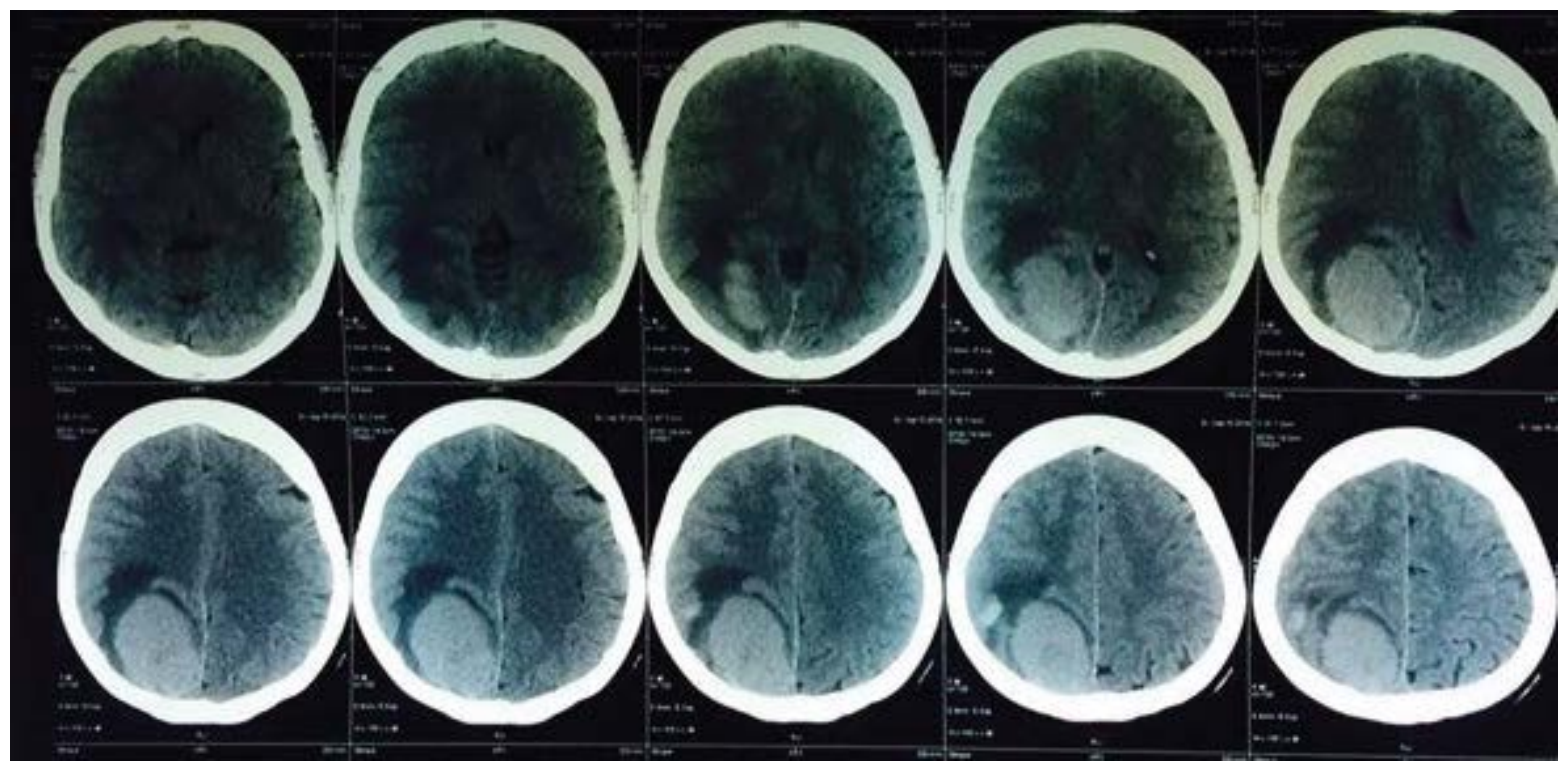

Figure 1a: Non enhanced axial CT brain showing a homogeneously hyperdense lesion in the right occipital lobe with peritumoral edema.
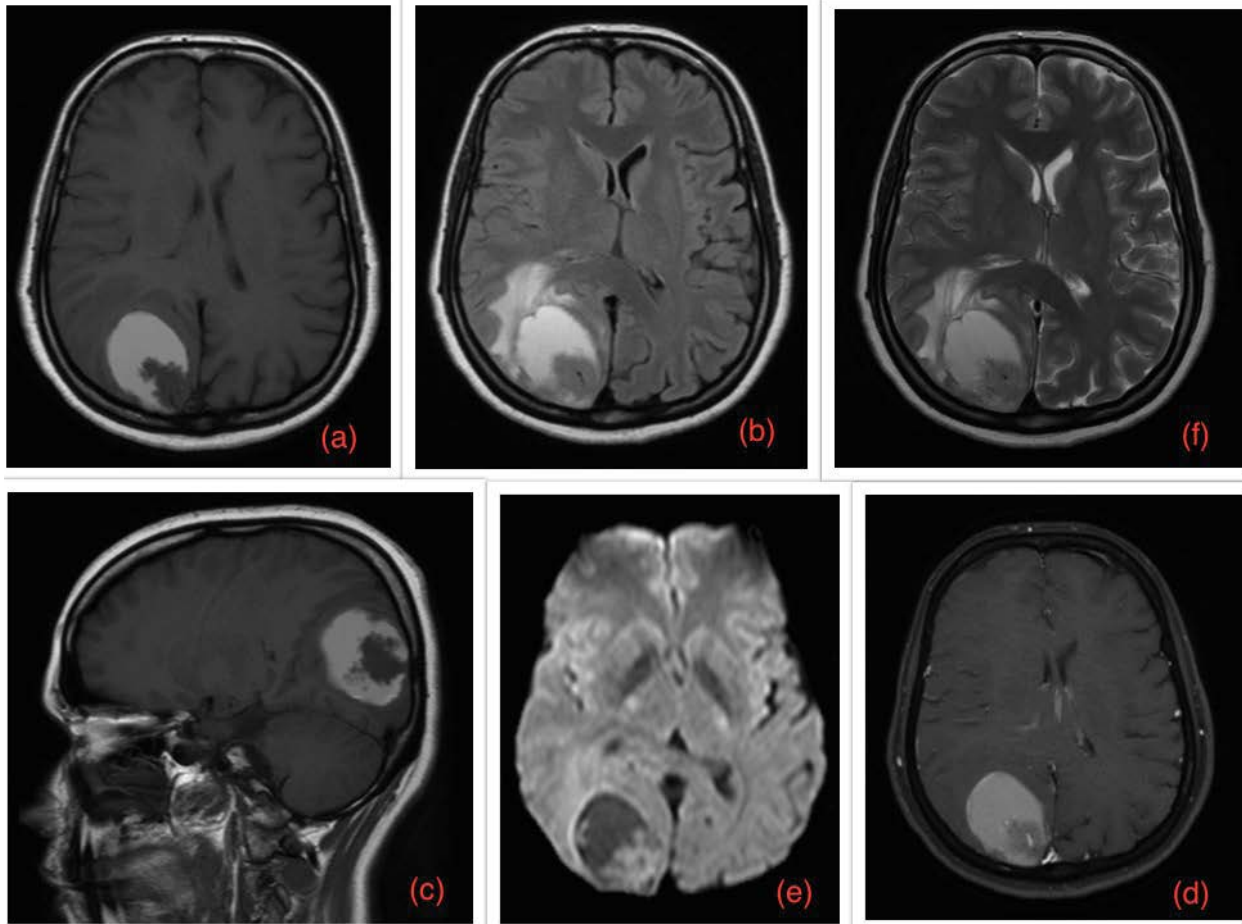

Figure 1b: Axial MRI showing a well-defined lesion in the right occipital lobe with heterogenous components and measuring $\sim 4.7 \times 3.4 \times 4.8 \mathrm{~cm}$. The peripheral part is hyperintense on T1 (b), T2 (c) and FLAIR (d) while the inner solid part is isointense to parenchyma on all these MRI sequences. The inner component shows restriction on DWI images (e) while showing mild enhancement on contrast images (f).

MRI appearances deviating from the classical ones pose a significant challenge, both for the clinician and radiologist. Appearance of T1 hyperintensity on MRI known as T1 shortening is contributed by various substances that include methemoglobin (in form of subacute blood), fat, high protein, calcium, melanin and other paramagnetic materials such as copper, manganese, iron $[10,11]$. The diagnosis can further be narrowed down based on additional signal characteristics on T2 weighted MRI and CT images. These have been briefly summarized in Table 1. Of these, either subacute blood containing methemoglobin or melanin are the only reported possibilities in metastasis, either in form of hemorrhagic ones (most commonly from kidney or thyroid) or a metastatic melanoma [10].

No reports of a high protein containing metastatic lesion can be found in literature till date. In our case, the lesion showed all possible features of high protein content-homogeneous mild hyperdensity on $\mathrm{CT}$, hyperintensity on both T1 and T2WI. The parenchymal lesions with high protein content reported are colloid cyst, craniopharyngioma, Rathke cleft cyst [10]. Rarely, tumours such as ependymoma with cystic 
Citation: Prasad GL, Nair R, Vasudevan G (2015) Brain Metastasis with High Protein Content- An Extremely Rare Imaging Feature. J Neurol Disord 3: 208. doi:10.4172/2329-6895.1000208

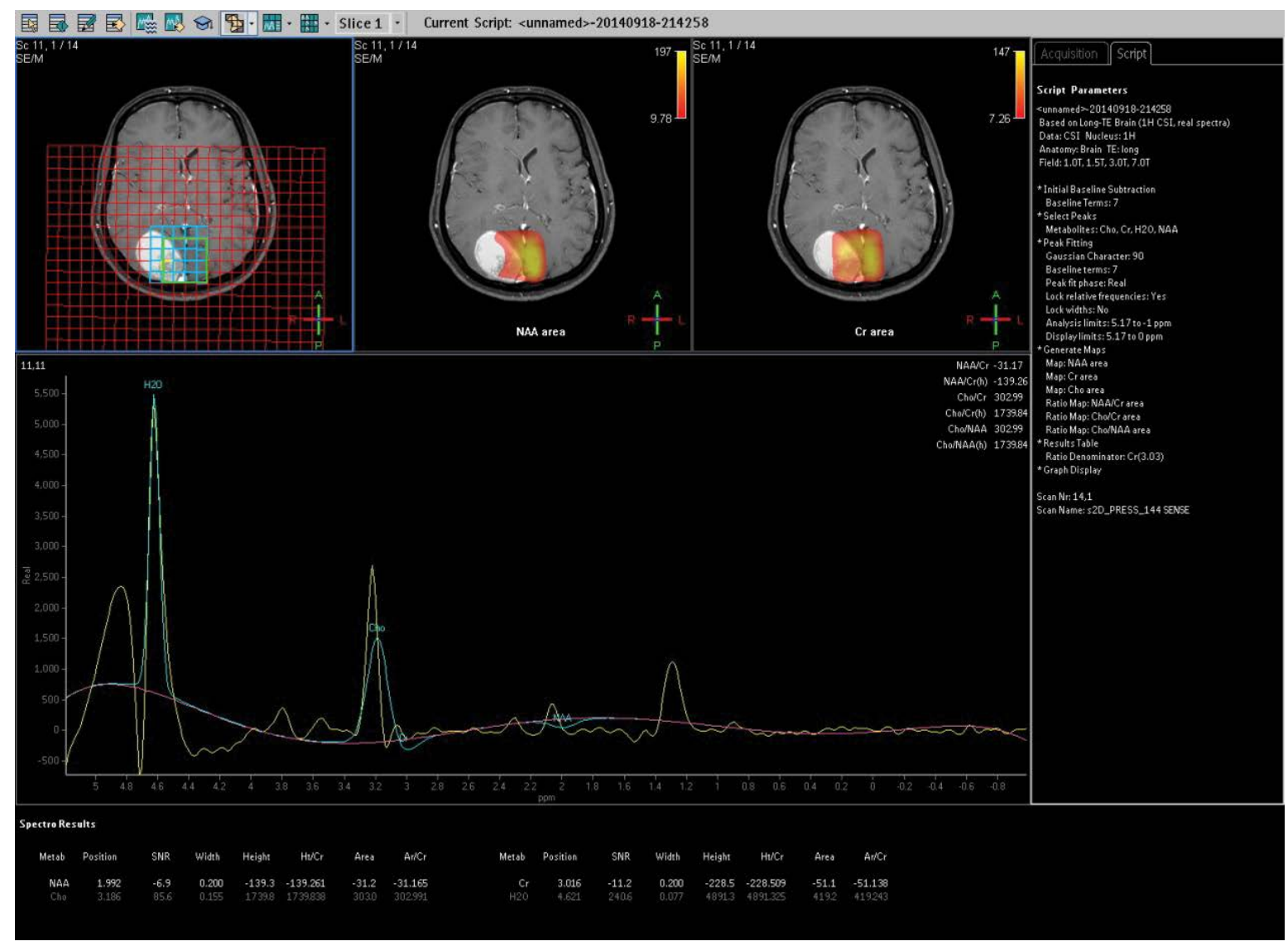

Figure 2: Intratumoral MR spectroscopy showing low Cho/Cr ratio with perilesional low NAA/Cho ratio $(a, b)$.

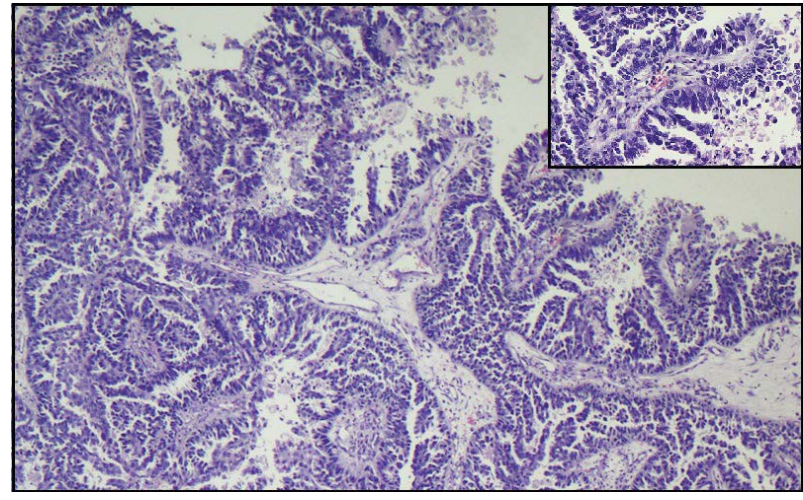

Figure 3: Photomicrograph showing metastatic papillary adenocarcinoma (H\&E, 4X), Inset showing fibrovascular core lined by pseudostratified malignant columnar cells (H\&E, 20X)

\begin{tabular}{|c|c|c|c|c|}
\hline Substance & CT & T1 WI & T2 WI & Lesions \\
\hline Methemoglobin & Hyper/iso & Hyper & $\begin{array}{c}\text { Hyper/ } \\
\text { hypo (extra/ } \\
\text { intracellular) }\end{array}$ & $\begin{array}{c}\text { Hemorragic tumours (a fluid } \\
\text { level is commonly seen), } \\
\text { hematoma, cavernomas, } \\
\text { thrombi }\end{array}$ \\
\hline Fat & Hypo & Hyper & Hyper & $\begin{array}{c}\text { Lipomas, teratomas, dermoids, } \\
\text { lipomatous neoplasm }\end{array}$ \\
\hline High protein & Hyper & Hyper & $\begin{array}{c}\text { Hyper/ rarely } \\
\text { hypo }\end{array}$ & $\begin{array}{c}\text { Colloid cyst, Rathke cleft cyst, } \\
\text { Craniopharyngioma }\end{array}$ \\
\hline Melanin & Hyper & Hyper & Hypo & Melanoma, melanocytoma \\
\hline
\end{tabular}

Table 1: List of the common T1 hyperintense producing substances and their signal intensities on various imaging sequences component, atypical teratoid rhabdoid tumour or choroid plexus carcinoma have also been described to have a machine-oil fluid with high protein content $[12,13]$. Also, the intra-tumoural $\mathrm{Cho} / \mathrm{Cr}$ was low with peri-lesional low NAA/Cho levels, which were suggestive more of a metastatic lesion. After ruling out all differentials, with the occipital lobe location and these imaging features, metastasis and an ependymoma with cyst were considered as possibilities. The contents of such tumours could be thick and gelatinous as in colloid cyst or machinery oil like as in a craniopharyngioma $[12,13]$. In our case, we found the typical machinery oil-like fluid within the metastatic tumour. Ours is the first case of a metastatic lesion possessing such features. A thorough work-up for primary tumour was performed even after surgery but was negative. Finally, she was referred for radiation oncology and is presently undergoing whole brain fractionated radiotherapy.

Prognosis of brain metastasis is usually poor [4]. Also, Karnosfky performance score is a robust predictor of survival and functional quality of life [14]. It has been reported that many patients with adenocarcinoma of unknown primary site have widespread metastases and poor performance status at the time of diagnosis. The outlook for most of these patients is poor, with median survival of 4 to 6 months. The role of systemic chemotherapy in addition to definitive local therapy is not clearly defined; however, patients with poorly differentiated adenocarcinoma may benefit from platinum-based therapy [14]. However, in our case, no other metastasis could be identified. Therefore, with such tumour peculiarities and a very short follow-up, it remains to be known the behavior of this tumour and the prognosis of our patient in the long-term. 
Citation: Prasad GL, Nair R, Vasudevan G (2015) Brain Metastasis with High Protein Content- An Extremely Rare Imaging Feature. J Neurol Disord 3: 208. doi:10.4172/2329-6895.1000208

\section{Conclusions}

We report the first case of a metastatic papillary adenocarcinoma with high protein content, based on imaging and per-operative features. The outlook for adenocarcinoma is relatively poor. The longterm prognosis of solitary metastatic papillary adenocarcinoma with unknown primary and above mentioned imaging peculiarities is yet to be known.

\section{References}

1. Hoover JM, Morris JM, Meyer FB (2011) Use of preoperative magnetic resonance imaging $\mathrm{T} 1$ and $\mathrm{T} 2$ sequences to determine intraoperative meningioma consistency. Surgical Neurology International 2: 142

2. Westphal M, Heese O, de Wit M. (2003) Intracranial metastases: therapeutic options. Ann Oncol 14 (Supplement 3): Siii4-S10.

3. Posner JB (1992) Management of brain metastases. Rev Neurol (Paris) 148 477-487

4. Norden AD, Wen PY, Kesari S (2005) Brain metastases. Curr Opin Neurol 18: $654-661$.

5. Delattre JY, Krol G, Thaler HT, Posner JB (1988) Distribution of brain metastases. Arch Neurol 45: 741-744.

6. Hwang TL, Close TP, Grego JM, Brannon WL, Gonzales F (1996) Predilection of brain metastasis in gray and white matter junction and vascular border zones. Cancer 77: 1551-1555.
7. Taphoorn MJ, Heimans JJ, Kaiser MC, de Slegte RG, Crezee FC, et al. (1989) Imaging of brain metastases. Comparison of computerized tomography (CT) and magnetic resonance imaging (MRI). Neuroradiology 31: 391-395.

8. Fink KR, Fink JR (2013) Imaging of brain metastases. Surg Neurol Int 4: S209-219.

9. Bulakbasi N, Kocaoglu M, Ors F, Tayfun C, Uçöz T (2003) Combination of single-voxel proton MR spectroscopy and apparent diffusion coefficient calculation in the evaluation of common brain tumors. AJNR Am J Neuroradio 24: $225-233$.

10. Ginat DT, Meyers SP (2012) Intracranial lesions with high signal intensity on T1-weighted MR images: differential diagnosis. Radiographics 32: 499-516.

11. Sijens PE, Knopp MV, Brunetti A, Wicklow K, Alfano B, et al. (1995) 1H MR spectroscopy in patients with metastatic brain tumors: a multicenter study. Magn Reson Med 33: 818-826.

12. Salunke P, Sahoo SK, Madhivanan K, Radotra BD (2014) A typical radiological presentation in a case of choroid plexus carcinoma. Surg Neurol Int 5: 63 .

13. Ahmadi J, Destian S, Apuzzo ML, Segall HD, Zee CS (1992) Cystic fluid in craniopharyngiomas: MR imaging and quantitative analysis. Radiology 182 783-785.

14. Hainsworth JD, Greco FA (2001) Adenocarcinoma of Unknown Primary Site In: Kufe DW, Pollock RE, Weichselbaum RR, et al., (eds.) (6thedn.), HollandFrei Cancer Medicine. 